\title{
The importance of cognitive flexibility and flexibility in coping with stress for the quality of life in inflammatory bowel disease patients during biological therapy. A preliminary report
}

\author{
Agata Rudnik ${ }^{1,2}$, Grażyna Piotrowicz², Małgorzata A. Basińska3 ${ }^{3}$ Vahid Rashedi 4,5 \\ ${ }^{1}$ Institute of Psychology, University of Gdansk, Gdansk, Poland \\ ${ }^{2}$ Department of Gastroenterology, Independent Public Health Care of the Ministry of the Internal Affairs, Gdansk, Poland \\ ${ }^{3}$ Department of Clinical Psychology, Kazimierz Wielki University, Bydgoszcz, Poland \\ ${ }^{4}$ School of Behavioural Sciences and Mental Health (Tehran Institute of Psychiatry), Iran University of Medical Sciences, \\ Tehran, Iran \\ ${ }^{5}$ Iranian Research Centre on Aging, University of Social Welfare and Rehabilitation Sciences, Tehran, Iran
}

Gastroenterology Rev 2019; 14 (2): 121-128

DOI: https://doi.org/10.5114/pg.2018.81081

Key words: ulcerative colitis, Crohn's disease, quality of life, biological treatment, mindfulness.

Address for correspondence: Anna Rudnik PhD, Institute of Psychology, University of Gdansk, 4 Bażyńskiego St, 80-309 Gdansk, Poland, phone: +48661 410 141, e-mail: agata.rudnik@ug.edu.pl

\begin{abstract}
Introduction: There are studies demonstrating the relation between psychological factors and efficiency of treatment in the course of inflammatory bowel diseases (IBD).

Aim: To investigate the relation between cognitive flexibility and flexibility in coping with stress and the quality of life, as well as the sense of satisfaction with life, in patients undergoing the biological treatment.

Material and methods: The study group consisted of 33 adults who were diagnosed with Crohn's disease (CD) or ulcerative colitis (UC). The Flexibility in Coping with Stress Questionnaire (FCSQ-14), the Cognitive Flexibility Inventory (CFI), the Quality of Life SF36v2 Questionnaire, the Satisfaction with Life Scale (SWLS), and the socio-demographic questionnaire were used to gather the data.

Results: A total of 14 females and 19 males were included in this analysis. The results did not prove that the disease diagnosis (UC or CD) differed the level of psychological resources. However, older respondents achieved lower results in cognitive flexibility. A positive correlation was observed between both cognitive flexibility and flexibility in coping with stress and different dimensions of quality of life and satisfaction with life.

Conclusions: There is a relationship between both cognitive flexibility and flexibility in coping with stress and health-related quality of life. These factors can be considered to be a kind of resource that helps to cope with challenges posed by a chronic illness, including IBD. Therefore, it is significant to increase their level by applying tailored therapeutic methods.
\end{abstract}

\section{Introduction}

Inflammatory bowel disease (IBD), including almost of all Crohn's disease (CD) and ulcerative colitis (UC), is a chronic relapsing-remitting condition primarily affecting the gastrointestinal tract. The CD and UC patients may experience such symptoms as abdominal pain, diarrhoea, weight loss, gastrointestinal bleeding, fatigue, and psychological distress [1]. A chronic disease is considered to be a difficult situation, which one has to adapt to in an appropriate way. It is a source of stress because it is related to numerous cognitive, somatic, and emotional changes [2]. The exacerbation of illness is both influencing and being influenced by biological and psychosocial factors. This is why the ability to cope with the disease and adjust one's own coping strategies to its demands are so significant for patients [3]. Due to the development of medicine, improvements in therapies of inflammatory bowel diseases are constantly being introduced. However, administration of these 
drugs does not always lead to desirable effects, such as diminishing the inflammatory symptoms or disease remission. Biological treatment is an alternative therapy for IBD patients in whom conventional therapy (using e.g. corticosteroids and drugs that affect the immune response) has failed [4]. It is based on the reactions against the particles of the immunological system (cells, cytokines, cytokine receptors). This treatment method was first applied in the 1990s. The vast majority of drugs are monoclonal antibodies, oriented against the TNF- $\alpha$ particle - tumour necrosis factor- $\alpha$ [5]. The tumour necrosis factor- $\alpha$ antibodies TNF- $\alpha$ include: infliximab (so far, its efficiency has been confirmed by the largest number of clinical studies), adalimumab, and certolizumab [6]. As far as CD is concerned, there are also clinical studies being conducted, which are focused on other monoclonal antibodies, including the first nonanti-TNF - natalizumab, which is a recombinant humanised antibody that targets the $\alpha 4$-subunit of both $\alpha 4 \beta 1$ and $\alpha 4 \beta 7$ integrins [7]. Although the biological therapy gives huge chances of recovery, it can be associated with numerous side effects, which have not yet been described in full. The side effects include e.g. anaphylactic reactions, cardiac insufficiency, onset or progression of symptoms of tuberculosis, multiple sclerosis, or lymphoma [8]. When patients are warned about the risk, they are often afraid to undergo the treatment. It is frequently the case that the treatment is inefficient, and the patient feels disappointed that he/she lost another chance to fight with the disease symptoms and achieve remission. Despite all odds, the biological treatment is very promising for many patients. It is a chance, a new opportunity, which gives hope, but also brings doubts and worries [2]. The patients frequently call it 'their last resort', but, on the other hand, they worry about its side effects and fear disappointment. During treatment many of them experience ups and downs, which makes it increasingly important to provide this group of patients with appropriate psychological counselling. It is possible, for example, by getting to know their psychological resources and checking whether their level is related to the quality of life of the patients. Such resources include cognitive flexibility and flexibility in coping with stress. Both the former and the latter are relatively new constructs, which allow assessment of the ability to cope with a change in life and adapt to new conditions. These factors, considered to be psychological resources, seem particularly important in coping with a chronic illness, which brings many challenges (e.g. its unpredictability, changes in treatment, or moments of disappointment). Cognitive flexibility is defined by Martin and Rubin (1995) as consisting of of three elements: awareness of the fact that the given situation is related to different available alternatives and options, the will to be flexible and open to adapt to situations and challenges that come along, as well as self-efficacy and belief in being adaptive and flexible. More cognitively flexible people can acknowledge the need for possible behavioural adaptation based on situational factors. Moreover, they are willing to try some new ways of communication, for example, to cope with unfamiliar situations and to adjust their behaviour to meet circumstantial needs [9]. The role of self-efficacy as a part of cognitive flexibility is also emphasised. It can be concluded that even if people are aware of alternative modes of behaviour in the given situation and they want to be flexible, they also have to believe that they can achieve the desired behaviour [10]. Individuals featured by high coping flexibility in stressful situations, which are yet controllable, usually apply primary approach strategies, such as problem solving. In order to cope with situations that they cannot control, they apply secondary approach strategies, such as meditation or relaxation. Studies have confirmed that flexible coping enhances well-being and health-related quality of life [11, 12]. Thus, on the basis of the quoted literature, we put forward the main hypothesis that patients living with IBD and during biological treatment, who present a higher level of cognitive flexibility and flexibility in coping with stress, obtain better results in terms of quality of life and a sense of satisfaction with life. People suffering from IBD cope with many difficulties on an everyday basis. Among others, these include symptoms impeding functioning, such as stomach ache, chronic fatigue, diarrhoea, and other unpleasant symptoms. The patients experience inconveniences in private, social, and professional life. This is why the need to provide them with psychological counselling is often emphasised. It should be based on in-depth recognition of both their needs and psychological resources, which could help the patients increase their quality of life and the level of satisfaction with life [13].

\section{Aim}

There are many studies demonstrating the relation between psychological factors and efficiency of treatment in the course of inflammatory bowel disease. We decided to investigate the relation between psychological factors (resources) such as cognitive flexibility and flexibility in coping with stress and the quality of life, as well as the sense of satisfaction with life in patients undergoing biological treatment, because there are few studies conducted on groups of patients undergoing this type of therapy. It seems particularly significant when the administered therapy evokes hope but also numerous worries. An appropriate level of psychological 
resources allows the challenges posed by illness to be overcome and even helps to increase the effectiveness of treatment.

\section{Material and methods}

The study group consisted of 33 adults $(N=33)$, 14 women and 19 men, who were diagnosed with Crohn's disease (18) or ulcerative colitis (15). All persons were in the course of biological treatment and were taking at least the third dosage of the drug. None of them was diagnosed with another chronic and life-threating disease or mental illness. The average age was $35.3 \pm 13$ years. The average duration of the disease was $8.5 \pm 6.9$ years. The demographic characteristics of the patients are presented in Table I.

The following research methods were used: the Flexibility in Coping with Stress Questionnaire - FCSQ-14 (Basińska et al., 2018) [14, 15], the Cognitive Flexibility Inventory, CFI, (Dennis, Vander Wal, 2010) - Polish adaptation [16]; the Quality of Life SF-36v2 Questionnaire - Polish version; the Satisfaction with Life Scale SWLS (Diener, Emmons, Larson, Griffin, 1985) - Polish adaptation (Juczyński, 2001); and the author's own questionnaire to collect the demographic data. The FCSQ-14 questionnaire demonstrates the ability of a person to modify the applied strategies of coping with stress depending on the current circumstances. The questionnaire consists of 14 statements assessed by respondents by selecting one of the following responses: 0 - never, 1 - sometimes, 2 - often, or 3 - always. The higher the score, the more flexible the person is in their coping [14, 15]. The obtained results are assigned to three scales: repertoire (the higher the result, the greater the range of behavioural and cognitive coping strategies and the ability to adapt them to the current situation), changeability (investigates the ability to change the coping strategy depending on the situation), and reflexivity (measures the ability to reflect on the applied strategies, their efficiency, and usefulness). The Cognitive Flexibility Inventory allows us to describe the ability of an individual to adapt to a difficult situation and replace maladaptive thoughts with a more adaptive way of thinking. The respondent obtains here an overall result and results in two subscales: control (perceiving a difficult situation as possible to control) and alternatives, which stands for the ability to develop many alternative solutions of a difficult situation) [16]. The Quality of Life SF-36v2 Questionnaire allows us to determine the quality of life of patients in terms of eight dimensions: vitality, physical functioning, bodily pain, general health perceptions, physical role functioning, emotional role functioning, social role functioning, mental health, and additionally a mental component sum-
Table I. Demographic characteristics of patients

\begin{tabular}{|c|c|c|}
\hline Parameter & Mean & $\begin{array}{l}\text { Standard } \\
\text { deviation }\end{array}$ \\
\hline \multirow[t]{2}{*}{ Age } & 35.3 & 13.0 \\
\hline & $N$ & $\%$ \\
\hline \multicolumn{3}{|l|}{ Sex: } \\
\hline Female & 14 & 42.4 \\
\hline Male & 19 & 57.6 \\
\hline \multicolumn{3}{|l|}{ Diagnosis: } \\
\hline Crohn's disease & 18 & 54.5 \\
\hline Ulcerative colitis & 15 & 45.5 \\
\hline \multicolumn{3}{|l|}{ Time passed after diagnosis: } \\
\hline Up to 5 years & 13 & 39.4 \\
\hline $6-10$ years & 8 & 24.24 \\
\hline $11-15$ years & 6 & 18.18 \\
\hline More than 16 years & 6 & 18.18 \\
\hline \multicolumn{3}{|l|}{ Marital status: } \\
\hline Single & 8 & 24.24 \\
\hline Married & 8 & 24.24 \\
\hline In an informal relationship & 13 & 39.4 \\
\hline After divorce (not in a relationship) & 2 & 6.06 \\
\hline After divorce (in a relationship) & 1 & 3.03 \\
\hline Widow/widower & 1 & 3.03 \\
\hline \multicolumn{3}{|l|}{ Number of children: } \\
\hline None & 18 & 54.5 \\
\hline One & 6 & 18.18 \\
\hline Two & 7 & 20.96 \\
\hline Three & 2 & 6.06 \\
\hline Four and more & 0 & 0 \\
\hline \multicolumn{3}{|l|}{ Education: } \\
\hline Less than high school & 1 & 3.03 \\
\hline High school & 17 & 51.52 \\
\hline Vocational/technical school & 3 & 9.09 \\
\hline Some collage & 6 & 18.18 \\
\hline College graduate & 6 & 18.18 \\
\hline Postgraduate degree or higher & 0 & 0 \\
\hline \multicolumn{3}{|l|}{ Professional activity: } \\
\hline Professionally active & 19 & 57.6 \\
\hline On pension & 7 & 20.96 \\
\hline Unemployed & 4 & 12.35 \\
\hline Other (e.g. student) & 3 & 9.09 \\
\hline \multicolumn{3}{|l|}{ Place of residence: } \\
\hline Village & 8 & 24.24 \\
\hline Small town (up to 50,000 inhabitants) & 6 & 18.18 \\
\hline Town (50,000-200,000 inhabitants) & 3 & 9.09 \\
\hline City (more than 200,000 inhabitants) & 16 & 48.49 \\
\hline
\end{tabular}


mary and physical component summary. The higher the scale result, the better the health-related quality of life (the lower the score, the greater the disability) [17]. The measurement result in the SWLS scale is a general indicator of the sense of satisfaction with life. The higher the result, the higher the assessed level of satisfaction with life of an individual [18]. All of the applied methods are reliable, standardised, and validated.

\section{Statistical analysis}

The statistical analyses were conducted using SPSS Statistic v25 software and OPTUM PRO CoRE software. All respondents were also informed about the aim of the research and participated in it voluntarily. All of them also received appropriate psychological counselling. The research was conducted from February to May 2018 in the Department of Gastroenterology at the Independent Public Health Care of the Ministry of the Internal Affairs in Gdansk, Poland.

\section{Ethical considerations}

The study received a positive opinion from the Ethical Committee at the Institute of Psychology at the University of Gdansk (no. 2/2018). All respondents consented in writing to take part in the study. The study procedure guaranteed the respondents full anonymity. All of them were informed about the aim of the research and could withdraw from the study at any time or refuse to take part in it. They received the contact data of the researcher, whom they could reach to get feedback and information about the obtained results. All respondents were also ensured appropriate psychological counselling, if they needed such, and two pa-

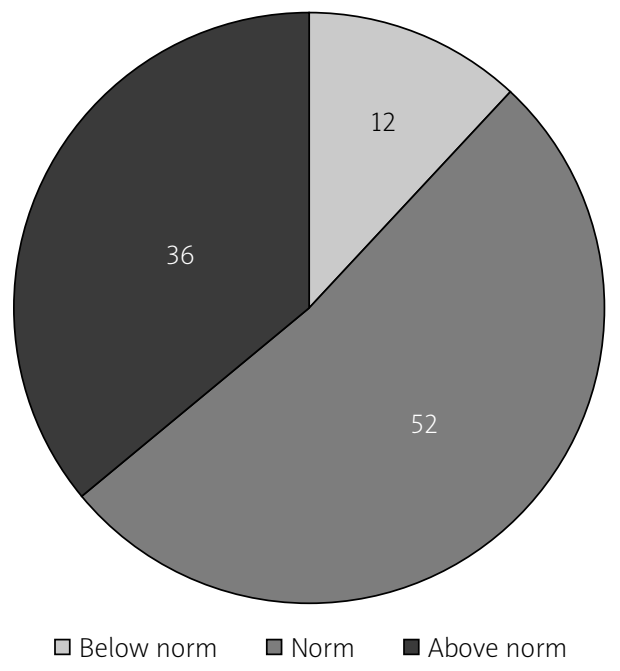

Figure 1. Quality of life of respondents - physical component summary (PCS) tients continue to attend psychological sessions at the gastroenterological ward.

\section{Results}

The physical component summary (PCS) and mental component summary (MCS) aggregate the scores of the SF-36 scales. In the majority of the patients both the PCS and MCS could be classified as 'normal' (42\% and $52 \%$, respectively). However, $36 \%$ of the respondents obtained results below the norm in the case of the physical component, and $39 \%$ of them in the case of the mental component. These findings are shown in Figures 1 and 2. This report was based on normative data from the QualityMetric 2009 General Population Sample [17].

The study did not prove that the disease diagnosis (UC or CD) affected the level of cognitive flexibility or flexibility in coping with stress (Table II). Sex did not affect the scale results either (Table III).

No relation between the duration of the disease and flexibility in coping with stress or cognitive flexibility was determined. However, a negative correlation between the cognitive flexibility-scale alternatives and age was observed (older respondents achieved lower results in that scale). The results are presented in Table IV.

A strong positive correlation $(p<0.01)$ was observed between cognitive flexibility and lower sense of limitation imposed by physical or emotional problems in everyday functioning (physical role functioning and emotional role functioning), as well as between cognitive flexibility and the sense of satisfaction with life. Another strong positive correlation $(p<0.01)$ was observed between the cognitive flexibility-scale control

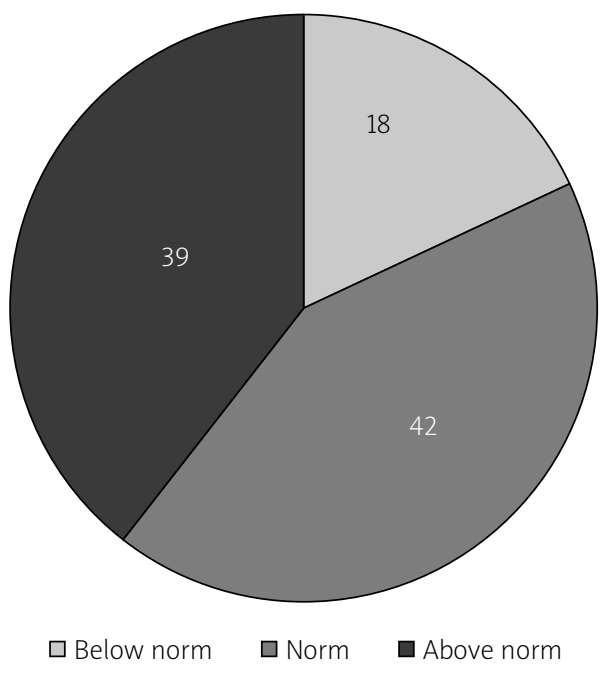

Figure 2. Quality of life of respondents - mental component summary (MCS) 
Table II. $T$-tests in which the grouping variable is the diagnosis of inflammatory bowel disease

\begin{tabular}{|c|c|c|c|c|c|c|c|c|c|}
\hline \multirow[t]{2}{*}{ Variables } & \multicolumn{2}{|c|}{$C D$} & \multicolumn{2}{|c|}{ UC } & \multirow[t]{2}{*}{$t$} & \multirow[t]{2}{*}{$\mathrm{d} f$} & \multirow[t]{2}{*}{$P$-value } & \multirow{2}{*}{$\begin{array}{c}F \\
\text { Variances }\end{array}$} & \multirow{2}{*}{$\begin{array}{c}P \text {-value } \\
\text { Variances }\end{array}$} \\
\hline & Mean & $\begin{array}{l}\text { Standard } \\
\text { deviation }\end{array}$ & Mean & $\begin{array}{l}\text { Standard } \\
\text { deviation }\end{array}$ & & & & & \\
\hline FCSQ_Reflexivity & 5.83 & 2.01 & 6.40 & 1.72 & -0.86 & 31 & 0.40 & 1.36 & 0.57 \\
\hline FCSQ_Changeability & 11.78 & 3.30 & 11.40 & 2.90 & 0.34 & 31 & 0.73 & 1.30 & 0.63 \\
\hline FCSQ_Repertoire & 9.44 & 2.57 & 8.73 & 2.52 & 0.80 & 31 & 0.43 & 1.04 & 0.95 \\
\hline $\mathrm{CFI}$ & 6.56 & 1.95 & 5.47 & 2.00 & 1.58 & 31 & 0.12 & 1.05 & 0.91 \\
\hline CFI_Control & 7.89 & 2.59 & 7.00 & 2.42 & 1.01 & 31 & 0.32 & 1.14 & 0.81 \\
\hline CFI_Alternatives & 5.50 & 1.29 & 5.46 & 2.10 & 1.51 & 31 & 0.14 & 2.62 & 0.06 \\
\hline
\end{tabular}

CD - Crohn's disease, UC - ulcerative colitis, FCSQ_Reflexivity Scale - Coping Flexibility - Reflexivity Scale, FCSQ_Changeability - Coping Flexibility Changeability Scale, FCSQ_Repertoire - Coping Flexibility - Repertoire Scale, CFI - Cognitive Flexibility, CFI_Control-Cognitive Flexibility - Control Scale, CFI_Alternatives - Cognitive Flexibility - Alternatives Scale.

Table III. T-tests in which the grouping variable is gender

\begin{tabular}{|c|c|c|c|c|c|c|c|c|c|}
\hline \multirow[t]{2}{*}{ Variables } & \multicolumn{2}{|c|}{ Female } & \multicolumn{2}{|c|}{ Male } & \multirow[t]{2}{*}{$t$} & \multirow[t]{2}{*}{$\mathrm{d} f$} & \multirow[t]{2}{*}{$P$-value } & \multirow{2}{*}{$\begin{array}{c}F \\
\text { Variances }\end{array}$} & \multirow{2}{*}{$\begin{array}{c}P \text {-value } \\
\text { Variances }\end{array}$} \\
\hline & Mean & $\begin{array}{l}\text { Standard } \\
\text { deviation }\end{array}$ & Mean & $\begin{array}{l}\text { Standard } \\
\text { deviation }\end{array}$ & & & & & \\
\hline FCS_Reflexivity & 6.14 & 2.18 & 6.05 & 1.68 & -0.13 & 31 & 0.89 & 1.67 & 0.31 \\
\hline FCS_Changeability & 10.92 & 3.08 & 12.11 & 3.07 & 1.10 & 31 & 0.29 & 1.00 & 0.97 \\
\hline FCS_Repertoire & 8.36 & 2.44 & 9.68 & 2.52 & 1.52 & 31 & 0.14 & 1.07 & 0.92 \\
\hline CF & 5.71 & 1.94 & 6.32 & 2.08 & 0.84 & 31 & 0.41 & 1.15 & 0.81 \\
\hline CF_Control & 7.00 & 2.94 & 7.84 & 2.17 & 0.95 & 31 & 0.35 & 1.83 & 0.23 \\
\hline CF Alternatives & 5.07 & 1.21 & 5.11 & 2.07 & 0.05 & 31 & 0.96 & 2.97 & 0.05 \\
\hline
\end{tabular}

FCS_Reflexivity Scale - Coping Flexibility - Reflexivity Scale, FCS_Changeability - Coping Flexibility - Changeability Scale, FCS_Repertoire - Coping Flexibility - Repertoire Scale, CF-Cognitive Flexibility, CF_Control - Cognitive Flexibility - Control Scale, CF_Alternatives-Cognitive Flexibility - Alternatives Scale.

Table IV. Coefficient of correlation between age elasticity and disease duration with flexibility in coping with stress and cognitive flexibility

\begin{tabular}{|c|c|c|c|c|c|c|}
\hline Variables & FCS_Reflexivity & FCS_Changeability & FCS_Repertoire & $\mathrm{CF}$ & CF_Control & CF_Alternatives \\
\hline Age & -0.16 & -0.19 & -0.06 & 0.30 & 0.01 & $-0.46^{\star *}$ \\
\hline Duration & -0.22 & -0.25 & -0.19 & 0.05 & 0.10 & -0.05 \\
\hline
\end{tabular}

Age - patient's age, Duration - duration of the disease, FCS_Reflexivity Scale - Coping Flexibility - Reflexivity Scale, FCS_Changeability - Coping Flexibility - Changeability Scale, FCS_Repertoire - Coping Flexibility - Repertoire Scale, CF-Cognitive Flexibility, CF_Control-Cognitive Flexibility - Control Scale,

CF_Alternatives - Cognitive Flexibility - Alternatives Scale. ${ }^{* *} p<0.01$

and lower sense of limitation imposed by physical or emotional problems in performing life roles (both physical and emotional role functioning). Physical role functioning correlated positively $(p<0.05)$ with the cognitive flexibility-scale repertoire, cognitive flexibility, and cognitive flexibility scales: control and alternatives. The physical role functioning scale correlated with coping with stress-repertoire scale (similarly with general health). The lower sense of limitation in performing life roles conditioned by emotional problems - emotional role functioning - correlated with the FCSQ subscales (both changeability and repertoire). Mental health correlated with the cognitive flexibility component control. The mental component of the quality of life correlated positively with cognitive flexibility. In turn, the sense of satisfaction with life correlated $(p<0.05)$ with the following FCSQ scales: changeability and repertoire, and additionally with the cognitive flexibility component alternatives. No relation between reflexivity and factors comprising the quality of life or the sense of satisfac- 
Table V. Coefficients of correlation of flexibility in coping with stress and cognitive flexibility with components of quality of life and a sense of satisfaction with life

\begin{tabular}{lccccccccccc} 
Variables & PF & BP & RP & GH & VT & SF & RE & MH & MCS & PCS & SWL \\
\hline FCS_Reflexivity & 0.15 & 0.01 & 0.13 & 0.05 & 0.04 & 0.11 & 0.156 & -0.02 & 0.07 & 0.08 & 0.34 \\
\hline FCS_Changeability & 0.26 & 0.01 & 0.34 & 0.27 & 0.02 & -0.10 & $0.34^{*}$ & -0.01 & 0.08 & 0.19 & $0.39^{*}$ \\
\hline FCS_Repertoire & $0.47^{*}$ & 0.01 & $0.41^{*}$ & $0.41^{*}$ & 0.15 & 0.09 & $0.41^{*}$ & 0.12 & 0.21 & 0.25 & $0.39^{*}$ \\
\hline CF & $0.48^{*}$ & 0.12 & $0.50^{* *}$ & 0.29 & 0.25 & 0.31 & $0.51^{* *}$ & 0.32 & $0.39^{*}$ & 0.26 & $0.47^{* *}$ \\
\hline CF_Control & $0.39^{*}$ & 0.01 & $0.46^{* *}$ & 0.23 & 0.12 & 0.30 & $0.49^{* *}$ & $0.38^{*}$ & $0.40^{*}$ & 0.12 & 0.28 \\
\hline CF_Alternatives & $0.37^{*}$ & 0.19 & 0.31 & 0.25 & 0.23 & 0.23 & 0.31 & 0.13 & 0.21 & 0.30 & $0.44^{*}$
\end{tabular}

FCS_Reflexivity Scale - Coping Flexibility - Reflexivity Scale, FCS_Changeability - Coping Flexibility - Changeability Scale, FCS_Repertoire - Coping Flexibility - Repertoire Scale, CF-Cognitive Flexibility, CF_Control - Cognitive Flexibility - Control Scale, CF Alternatives - Cognitive Flexibility - Alternatives Scale, PF - Physical Functioning, BP - Bodily Pain, RP - Physical Role Functioning, GH - General Health Perceptions, VT - Vitality, SF-Social Functioning, RE - Emotional Role Functioning, MH - Mental Health, MCS - Mental Component Summary, PCS - Physical Component Summary, SWL - Satisfaction with Life. * $p<0.05$, ${ }^{* *} p<0.01$

tion with life was demonstrated. The coefficients of the discussed correlations are presented in Table V.

\section{Discussion}

Many researchers emphasise the relation between the psychological factors and the aetiology and course of the IBD. In addition, the disease itself - CU and CD affects the mental state of the patient. Morys, Kaczów$k a$, and Jezewska indicate that 'patients with IBD suffer elevated or high sense of anxiety'. However, their study did not reveal differences among personality factors in a group of patients with the UC and CD [19]. The study conducted by Graff et al. on a group of 388 individuals with IBD also did not demonstrate a difference between psychological profiles of ulcerative colitis and Crohn's disease participants [20]. Similarly, it was demonstrated in our research that the disease diagnosis did not differentiate results obtained in the flexibility in coping with stress and cognitive flexibility scales. Therefore, we decided to investigate the relation of these variables with the quality of life and the sense of satisfaction with life jointly for both groups of patients. As Mikocka-Walus et al. emphasised in their review, they were unable to examine gender differences in anxiety and depression comorbidity because there is currently no research available that would unequivocally indicate the female/ male breakdown of the results [21]. As it was presented in our study, sex did not differentiate the level of psychological factors either, e.g. the level of cognitive flexibility or flexibility in coping with stress. However, the analyses we conducted revealed a negative correlation between cognitive flexibility and age. Eldesouky and English asked the question: another year older, another year wiser? Their study, conducted on a group of 136 married couples aged 23-85 years, opposes the common assumption that the elder respondents usually use adaptive strategies more often than younger adults. Alternatively, their findings suggest that the same group may be 'more consistent in their emotion regulation patterns across situations, potentially suggestive of less flexibility' [22]. A systematic review of the psychological correlates of adjustment outcomes in adults with inflammatory bowel disease, conducted by Jordan et al., revealed six main categories of factors, which comprised: personality traits, interpersonal traits, emotions and emotional control, stress and coping, IBD-related cognitions, and non-IBD-related cognitions. The authors suggest that these factors should be considered to be the intervention targets. They also emphasise that the most persistent relationship occurred between certain emotion-focused coping strategies and worse adjustment outcomes in the inflammatory bowel disease [23]. In the current study, fitting with this trend, we obtained results that unequivocally confirm our main hypothesis and show a strong positive correlation between flexibility in coping with stress and cognitive flexibility, and both the health-related quality of life (HRQL) and the sense of satisfaction with life. Our study also showed that the group that requires special support and care are the older patients. That is why their needs in the field of psychological resources should be met in a special way. The obtained analyses are confirmed by many other studies conducted on groups of patients with gastrointestinal diseases. Stanculete et al. revealed that coping strategies, as well as irrational beliefs, can be the mediators of HRQL impairments in irritable bowel syndrome [24]. In addition, Sherwin, Leary, and Henderson (2017) emphasised that patients with IBS, who catastrophised (catastrophising is defined here as a cognitive process - an inclination to focus on and magnify the value of an actual or anticipated painful stimulus) presented worse psychosocial and functional outcomes [25]. Interesting 
results were also presented by Luo et al., who revealed that individuals with ulcerative colitis, who demonstrated acceptance-resignation behaviour, assessed their quality of life as poor, and, moreover, such an attitude was the predictor of future hospitalisation because of a relapse. It was underlined in that publication that improving the medical coping behaviour in that group of patients by means of psychotherapy could be beneficial to attaining a better quality of life and disease control [26]. The question is then how to support patients during the biological treatment. Our study confirms that it is necessary to focus on such psychological factors as cognitive flexibility and flexibility in coping with stress. Oishi et al. demonstrated that a properly developed web-based training program using cognitive behavioural therapy enhanced cognitive flexibility and eased psychological distress among schoolteachers [27]. The Internet cognitive behavioural therapy is applicable to inflammatory bowel disease patients and can improve their quality of life. What is more, cognitive flexibility can be improved by an appropriate mindfulness-based cognitive therapy, which is confirmed, for example, by Shapero et al. [28]. The positive impact of the mindfulness-based exercises on the IBD patients was highlighted in the past by, for example, Ballou and Keefer [29]. What is more, Sollie et al. pointed out that mindfulness-based intervention for a group of patients with chronic fatigue syndrome increased not only their quality of life but also more adaptive coping [30]. Therefore, it seems justifiable to develop an appropriate therapeutic program for CD or UC patients during their biological treatment, in order to enhance their quality of life and hence make their attitude to the treatment more optimistic, which can also enhance its efficiency. On the other hand, we are aware of the challenges that the practical solutions based on the obtained results may bring. Among them are the time-consuming process of creating an appropriate mindfulness-based therapy program and finding specialists in this field.

What was not embraced by our study was the matter of how long a patient undergoes the biological therapy (the condition of taking part in this research project was taking at least the third dosage) or the type of the administered drug. In the future it would be worth including these factors in the analysis.

\section{Conclusions}

There is a relationship between such psychological factors as cognitive flexibility and flexibility in coping with stress. These factors can be considered to be a kind of resource to help to cope with challenges posed by a chronic illness, including the course of the inflammatory bowel disease. Therefore, it is important to increase their level by applying therapeutic methods tailored to the needs of the patient.

\section{Acknowledgments}

This study is a part of the project implemented at the University of Gdansk in cooperation with Independent Public Health Care of the Ministry of the Internal Affairs in Gdansk.

\section{Conflict of interest}

The authors declare no conflict of interest.

\section{References}

1. Farrell D, McCarthy G, Savage E. Self-reported symptom burden in individuals with inflammatory bowel disease. J Crohn's Colitis 2015; 10: 315-22.

2. Ziarko M. Zmaganie się ze stresem choroby przewlekłej [Coping with a stress of chronic illness]. Wydawnictwo Naukowe Wydziału Nauk Społecznych UAM [Scientific Publisher of the Faculty of Social Sciences of Adam Mickiewicz University]. Poznan 2014

3. Bernstein $\mathrm{CN}$. The brain-gut axis and stress in inflammatory bowel disease. Gastroenterol Clin North Am 2017; 4: 839-46.

4. Bernstein CN. Treatment of IBD: where we are and where we are going. Am J Gastroenterol 2015; 110: 114-26.

5. Schölmerich J, Huber G. Biological therapy in IBD. Dig Dis 2003; 21: 180-91.

6. Kawalec P, Mikrut A, Wiśniewska N, et al. Tumor necrosis factor-alpha antibodies (infliximab, adalimumab and certolizumab) in Crohn's disease: systematic review and meta-analysis. Arch Med Sci 2013; 9: 765-79.

7. Kurtovic J, Segal I. Recent advances in biological therapy for inflammatory bowel disease. Trop Gastroenterol 2004; 25: 9-14.

8. Hansel TT, Kropshofer $H$, Singer T, et al. The safety and side effects of monoclonal antibodies. Nature Rev Drug Discov 2010; 9: 325-38.

9. Martin MM, Rubin RB. A new measure of cognitive flexibility. Psychol Rep 1995; 76: 623-6.

10. Bandura A. Self-efficacy: towards a unifying theory of behavioural change. Am Psychol 1977; 33: 344-58.

11. Gan $Y$, Zhang Y, Wang $X$, et al. The coping flexibility of neurasthenia and depressive patients. Pers Individ Diff 2006; 40: 859-71.

12. Cheng C, Lau HPB, Chan MPS. Coping flexibility and psychological adjustment to stressful life changes: a meta-analytic review. Psychol Bull 2014; 140: 1582-607.

13. Lönnfors S, Vermeire S, Avedano L. IBD and health-related quality of life - discovering the true impact. J Crohn's Colitis 2014; 8: 1281-6.

14. Basińska MA, Sołtys M, Borzyszkowska, A. Personal resources resiliency and hope and flexibility in coping with stress among oncological patients. $32^{\text {nd }}$ Conference o the EHPS, Health Psychology Across the Lifespan: Uniting Research, Practice and Policy. 2018; Abstract Booklet. Ireland.

15. Grzankowska I, Kruczek A, Sołtys A, et al. The Flexibility Coping Questionnaire-14 (FCQ-14) in Polish adolescents. 32 ${ }^{\text {nd }}$ Con- 
ference of the EHPS, Health Psychology Across the Lifespan: Uniting Research, Practice and Policy. 2018; Abstract Booklet. Ireland.

16. Piórowski K, Basińska MA, Piórowska A, et al. Adaptation of the cognitive flexibility inventory. Przegl Psychol 2017; 4: 617-48.

17. Maruish ME (ed.) User's manual for the SF-36v2 Health Survey ( $3^{\text {rd }}$ ed.). Lincoln, RI: QualityMetric Incorporated 2011.

18. Juczyński Z. Narzędzia Pomiaru w Promocji i Psychologii Zdrowia. Wydanie drugie [Measurement tools in health promotion and in health psychology. Second edition]. Pracownia Testów Psychologicznych Polskiego Towarzystwa Psychologicznego, [Laboratory of Psychological Tests of the Polish Psychological Association]. Warsaw 2012

19. Morys J, Kaczówka A, Jeżewska, M. Assessment of selected psychological factors in patients with inflammatory bowel disease. Gastroenterol Rev 2015; 11: 47-53.

20. Graff LA, Walker JR, Lix L, et al. The relationship of inflammatory bowel disease type and activity to psychological functioning and quality of life. Clin Gastroenterol Hepatol 2006; 4: 1491-501.

21. Mikocka-Walus A, Knowles SR, Keefer L, et al. Controversies revisited: a systematic review of the comorbidity of depression and anxiety with inflammatory bowel diseases. Inflamm Bowel Dis 2016; 22: 752-62.

22. Eldesouky L, English T. Another year older, another year wiser? Emotion regulation strategy selection and flexibility across adulthood. Psychol Aging 2018; 33: 572.

23. Jordan C, Sin J, Fear NT, et al. A systematic review of the psychological correlates of adjustment outcomes in adults with inflammatory bowel disease. Clin Psychol Rev 2016; 47: 28-40.

24. Stanculete MF, Matu S, Pojoga C, et al. Coping strategies and irrational beliefs as mediators of the health-related quality of life impairments in irritable bowel syndrome. J Gastrointest Liver Dis 2015; 24: 159-64.

25. Sherwin LB, Leary E, Henderson, WA. The association of catastrophizing with quality-of-life outcomes in patients with irritable bowel syndrome. Qual Life Res 2017; 26: 2161-70.

26. Luo H, Sun Y, Li Y, et al. Perceived stress and inappropriate coping behaviors associated with poorer quality of life and prognosis in patients with ulcerative colitis. J Psychosom Res 2018; 113: 66-71.

27. Oishi S, Takizawa T, Kamata N, et al. Web-based training program using cognitive behavioral therapy to enhance cognitive flexibility and alleviate psychological distress among schoolteachers: pilot randomized controlled trial. JMIR Res Protoc 2018; 7: e32.

28. Shapero BG, Greenberg J, Mischoulon D, et al. Mindfulness-based cognitive therapy improves cognitive functioning and flexibility among individuals with elevated depressive symptoms. Mindfulness 2018; 9: 1-13.

29. Ballou S, Keefer L. Psychological interventions for irritable bowel syndrome and inflammatory bowel diseases. Clin Transl Gastroenterol 2017; 8: e214.

30. Sollie K, Næss E, Solhaug I, et al. Mindfulness training for chronic fatigue syndrome: a pilot study. Health Psychol Rep 2017; 5: 240-50

Received: 29.10 .2018

Accepted: 19.11 .2018 